\title{
II. MITTEII.UNGEN
}

\section{Professor Dr. Dr.h.c. Hubert Hugo Hilf 80 Jahre}

Am 11. April 1973 feicrte Professor HILf, der von 1945 bis zu seiner Emeritierung im Jahre 1961 Leiter des Instituts für forstliche Arbeitswissenschaft (Iffa) der Bundestorschungsanstalt für Forst- und Holzwirtschaft in Reinbek war, bei guter Gesundheit seinen 80. Geburtstag.

H. H. Huf wurde 1928 zum o. Professor der Forstlichen Hochschule Eberswalde berufen und war von 1935 bis 1945 Rcktor dieser Hochschule.

In Lebenswerk von HrLF spielt die forstiche Arbeitswissenschaft, deren Entwicklung in erster Linie auf ihn zurückgeht, die wichrigste Rolle. Wie sehr er sich bis in die Gegenwart mit forst- und holzwirtschaflichen Fragen auseinandergesetzt hat, zeigt der Umstand, daß in den letzten fünf Jahren noch 25 Veröffentlichungen von ihm erschienen sind.

Eine fachliche Würdigung zu seinem 70. Geburtstag ist im 82. Jahrgang dieser Zeitschrift erschienen.

Für seinen weiteren Lebensabend seien dem Jubilar die herzlichsten Wünsche für Glück und Gesundheit ausgesprochen.

\section{KWF-Tagung 1973}

Das Kuratorium für Waldarbeit und Forstrechnik (KWF) hält seine 5. Arbeitstagung in der Zeit vom 7. bis 11. Mai 1973 in Trier ab. Das Thema lautet:

"Der Wald, der im Walde tätige Mensch und die Forsttechnik"

Tagungsprogramme und Anmeldeunterlagen können bei der Geschäftsführung des KWF in 6079 Buchschlag, Postfach, angefordert werden.

\section{Deutsche Pflanzenschutztagung}

Vom 1. bis 5. Oktober 1973 finder in Sturtgart die 39. Deursche PflanzenschutzTagung statt. Im Programm sind u. a. folgende Themenkreise vorgesehen:

Aktuelle Probleme aus dem Gebiet der Phytomedizin, Phytopharmakologie, Formulierung und Applikation von Pflanzenschutzmitteln, biotechnische Verfahren zur Sdiädlingsbekämpfung und anderes.

Anmeldung und Anfragen sind zu richten an die Biologische Bundesanstalt für Land- und Forstwirtschaft, 3300 Braunschweig, Messeweg 11/12. 